\title{
Comprensión de los efectos escolares en Sudáfrica a través del análisis multinivel: hallazgos de la TIMSS 2011
}

\section{L.D. Winnaar ${ }^{1}$, G. Frempong ${ }^{2}$ y R. Blignaut ${ }^{3}$}

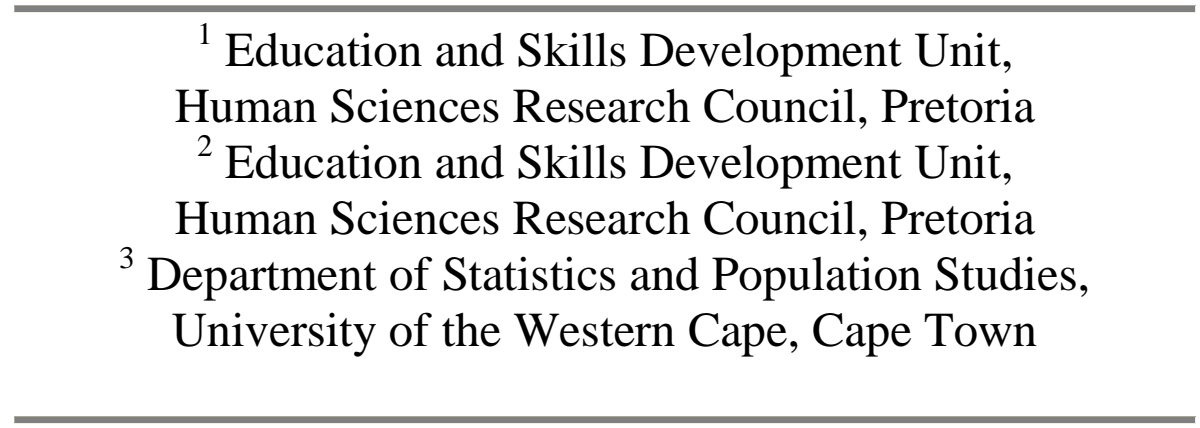

\section{Sudáfrica}

Correspondencia: Lolita Winnaar. 134 Pretorius Street, Pretoria, 0001. South Africa.

E-mail: LWinnaar@HSRC.ac.za

(C) Education \& Psychology I+D+i and Ilustre Colegio Oficial de la Psicología de Andalucía Oriental (Spain) 


\section{Resumen}

Introducción. Los estudios sobre el efecto de la escuela, por lo general, giran en torno a dos temas principales: la comprensión de los factores dentro de las escuelas que explican la variación en los niveles de logro entre los estudiantes, y las diferencias entre las escuelas respecto a la variación en el rendimiento de sus estudiantes. Dada la limitación de la investigación del efecto de la escuela en Sudáfrica, este trabajo explora cómo las diferentes características de los estudiantes y de la escuela están relacionadas con el rendimiento.

Método. Utilizando los datos del TIMSS Sudáfrica 2011 (TIMSS), esta investigación emplea el análisis multinivel para identificar los indicadores más importantes de las escuelas exitosas de Sudáfrica. El análisis inicial implica la partición de la variación total en los estudiantes según el nivel de logro en matemáticas, intra y entre escuelas. Esto nos permitió calcular el efecto aproximado de la escuela (en la medida en que las escuelas varían en los niveles de logro de sus estudiantes). El análisis de seguimiento consistió en añadir características escolares y de los estudiantes en los modelos multinivel para perfilar los estudiantes y las escuelas que tienen más éxito.

Resultados. El análisis multinivel indica que las diferencias en las escuelas representan alrededor del $62 \%$ de la variación en el nivel de logro de los estudiantes en matemáticas. Los estudiantes con más éxito son los que asisten a las escuelas que cuentan con recursos adecuados, tienen maestros que están satisfechos con sus condiciones de trabajo y que están especializados en el campo de la matemática. También muestran que la actitud de los estudiantes hacia las matemáticas tiene un impacto en su desempeño.

Discusión. Los resultados demuestran la necesidad de que los responsables de las políticas educativas de Sudáfrica sigan mejorando el ambiente de aprendizaje de las escuelas con menos recursos y también animen a los profesores a inculcar un sentido de valor y confianza en sí mismo en el aprendizaje de matemáticas y mejorar la preparación del profesorado.

Palabras clave: modelado jerárquico lineal, análisis multinivel, las matemáticas, la eficacia escolar, TIMSS. 


\title{
Understanding school effects in South Africa using multilevel analysis: findings from TIMSS 2011
}

\begin{abstract}
Introduction. Studies of school effect usually evolve around two major issues; understanding of the factors within schools that explain variation in students achievement levels, and how differences among schools account for the variation in their students' achievement levels. Given the limited studies of school effect research in South Africa, this paper explores how different characteristics of students and school are related to performance.

Method. Using the 2011 South African TIMSS (Trends in International Mathematics and Science Study) data, the study employs multilevel analysis to identify the most important indicators of successful schools in South Africa. The initial analysis entails the partitioning of the overall variation in students' achievement level in mathematics into within and between schools that allows us to estimate school effects (the extent to which schools make a difference in students' achievement levels). A follow-up analysis involves the addition of student and school characteristics in the multilevel models to profile students and schools that are most successful.

Results. The multilevel analysis indicated that differences in schools account for about $62 \%$ of the variation in students' achievement levels in mathematics. Students are more successfulwhen they attend schools that are adequately resourced, have teachers that are satisfied with their working conditions and that are specialised in the field of mathematics. The results also show that the attitude of the students toward mathematics has an impact on their performance.

Discussion. The findings demonstrate the need for the South African educational policy makers to continue improving the learning environment of less resourced schools and also encourage teachers to instil a sense value and self-confidence in learning mathematics and to improve teacher qualification.
\end{abstract}

Keywords: Hierarchical Linear Modeling, Multilevel analysis, mathematics, school effectiveness, TIMSS

Reception: 07/08/14 Initial acceptance: 10/23/14 Final acceptance: 03/10/15 


\section{Introducción}

Durante las últimas décadas, los datos de valoración y análisis a gran escala han desempeñado un rol importante en el desarrollo de la comprensión sobre los efectos de los centros de educación - la medida en la que un centro en específico incide en el aprendizaje. En años recientes, los modelos multinivel son los análisis estadísticos preferidos ya que estos modelos permiten a los investigadores modelar la estructura multinivel del sistema de escolarización, es decir, grupos de alumnos que forman clases que están agrupadas en centros. Sin embargo, en Sudáfrica, a pesar de la disponibilidad de los conjuntos de datos de gran escala que proveen oportunidades para los análisis de multinivel, solo unas pocas publicaciones de investigación han usado los modelos multinivel para analizar los efectos de los centros de educación.

Los conjuntos de datos sudafricanos disponibles y adecuados para el análisis multinivel incluyen estudios comparativos internacionales tales como el SACMEQ (Southern and Eastern African Consortium for Measuring Educational Quality, Consorcio para medir la calidad educativa de África Meridional y Oriental) y el TIMSS (Trends in International Mathematics and Science Study, Estudio de las tendencias en el estudio de las matemáticas y las ciencias). El análisis de los datos del SACMEQ al usar los modelos multinivel indicó que, en los 14 países subsaharianos que participaron en los estudios, los centros inciden de forma significativa en los niveles de logro de sus alumnos del $6^{\circ}$ curso (vea (Lee, Zuze, y Ross, 2005) y (Hungi y Thuku, 2010)). El análisis realizado por Hungi y Thuku (2010) reveló también que Sudáfrica, junto con Uganda, y Namibia, mostró la mayor variación entre los centros demostrando el grado en que los centros inciden en los resultados del aprendizaje de los alumnos y la necesidad de desarrollar un entendimiento sobre el efecto del centro en Sudáfrica.

\section{Marco conceptual de la eficacia del centro de educación}

El enfoque del estudio es para explicar el impacto de los factores contextuales relacionados con los alumnos, sus familias y los centros sobre la eficacia de los centros. Hay dos componentes amplios que definen los marcos conceptuales de la investigación sobre la eficacia del centro. El primero es el punto de vista sociológico, el cual abarca cuatro áreas principales, concretamente: la ecología, el ambiente, el sistema social y la cultura. El segundo marco, que es también el marco que usamos en este artículo, se basa en el modelo económico de la escolarización (Levin, 2001) que se compone de 3 dimensiones; los factores base 
(inputs) de la escolarización, el proceso educativo y los resultados (outputs) de la escolarización.

La figura 1 proporciona una ilustración del modelo conceptual económico de escolarización que muestra el proceso de educación que ocurre en tres niveles: el centro, el aula, y el alumno. Es importante notar que los tres niveles están interconectados o que son multinivel por naturaleza, lo que quiere decir que las actividades en un nivel reciben influencia de actividades de niveles superiores (Willms y Somer, 2001).

Las dos dimensiones que influyen en los resultados de la escolarización son los "factores base (inputs) de los centros", refiriéndose a factores como, por ejemplo, la estructura del centro, el tamaño del centro y su ubicación. También incluyen las características del alumno y los recursos físicos de los que disponen los profesores para realizar una enseñanza adecuada. Teniendo en cuenta estos factores, está claro que los "factores base de los centros" generalmente son factores que, , se dan directamente, y, por lo tanto, la falta o la abundancia de estos recursos es algo que se encuentra fuera del control de los centros. La otra dimensión es la de "los procesos y prácticas del centro de educación y del aula", y incluyen información como el clima social y académico del centro de educación, el plan de estudios, las prácticas para la enseñanza y la organización social. Ésta suele ser la dimensión de interés para la mayoría de los responsables de la política educativa ya que los elementos de los procesos y las prácticas están, en cierta medida, dentro del control del centro de educación y se refieren a temas tales como el estilo de la gestión.

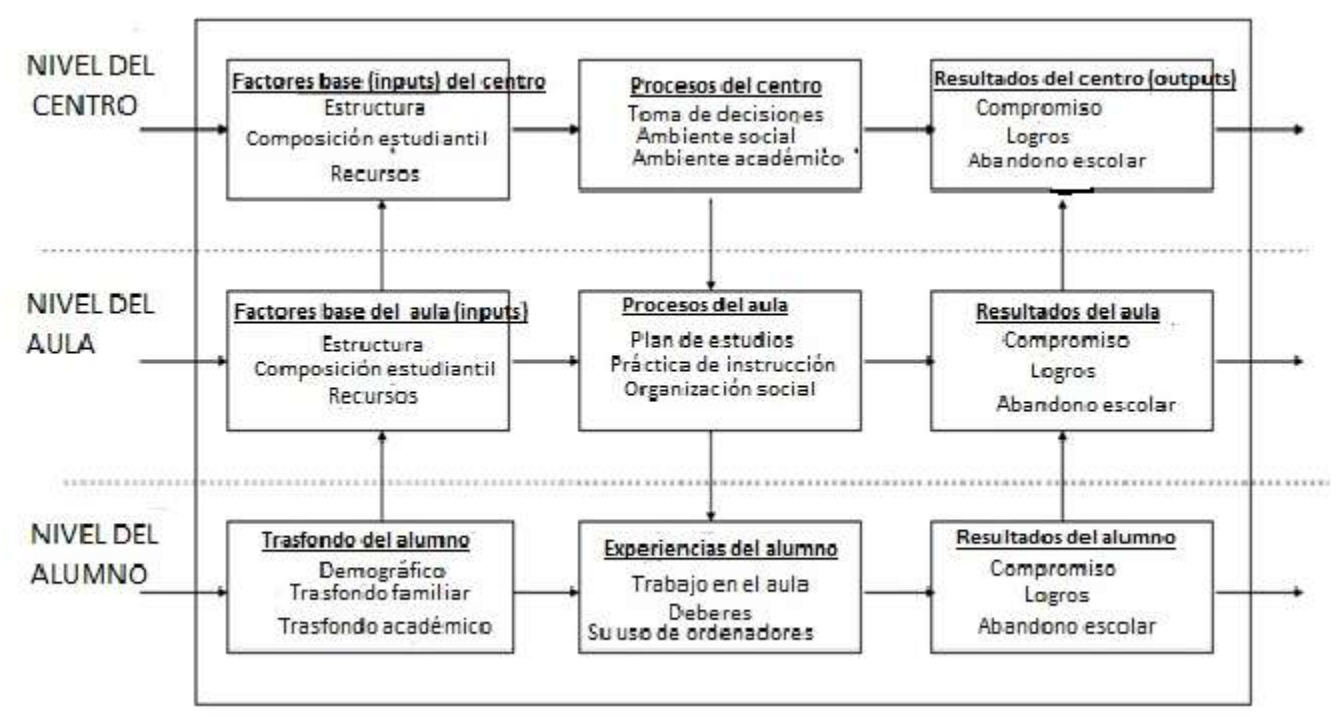

Figura 1. Un marco conceptual multinivel de la eficacia del centro (Rumberger y Palardy, 2004) 
El análisis de los datos de Sudáfrica en el TIMSS de 1995, a través de modelos multinivel, demostró que alrededor del 55 por ciento de la variación de los niveles de logro de los alumnos en matemáticas puede explicarse con las diferencias en la calidad de sus centros (vea Howie y Plomp, 2003) ). El análisis de Howie y Plomp (2003) mostró también que el dominio de los alumnos del idioma inglés es un determinante importante de su éxito en el aprendizaje de las matemáticas. Su análisis multinivel demostró que los aprendices tienen éxito en el aprendizaje de las matemáticas en las escuelas donde el profesor tiene más tiempo para trabajar y planificar la lección. El análisis de los resultados de datos del TIMSS de Sudáfrica entre el 1995 y el 2003 no mostró mejora significativa en el rendimiento promedio de matemáticas (Reddy, 2012); Sin embargo, parece haber una mejora significativa entre los ciclos del 2003 al 2011. Esta mejora significativa podría deberse a cambios en las políticas y en el plan de estudios implementados por el gobierno después de 1994 en un intento de rectificar la disparidad entre escuelas como legado del apartheid.

\section{Objetivo}

La pregunta es: ¿Cuáles son las políticas educativas sudafricanas que parecen marcar una diferencia en los niveles de logros de los alumnos? Este artículo tiene como objetivo, con el uso de modelos multinivel, describir centros exitosos que proporcionan entornos propicios para el aprendizaje de las matemáticas.

\section{Método}

Factores sobre el trasfondo del alumno y sobre el centro de educación

Las variables consideradas en el análisis o correspondían directamente a las encuestas de trasfondo, o se aunaban diferentes variables para medir un constructo subyacente. Para construir las escalas se utilizó la metodología de escala IRT (teoría de respuesta al ítem). Más información sobre la creación de la escala para todos los constructos del TIMSS se encuentra en la guía internacional del usuario de TIMSS (Joncas y Foy, 2013).

\section{Medidas de resultado (output)}

El TIMSS sigue un diseño de muestreo matriz lo que significa que los alumnos no contestaron todos los ítems. Por tanto, se usó la teoría de respuesta al ítem (IRT) para atribuir las puntuaciones de los alumnos y para crear 5 valores plausibles para cada alumno que se 
usaron en el análisis de modelos lineales jerárquicos (HLM) (P. Foy, Brossman, y Galia, 2011). Al aplicar la teoría de respuesta al ítem (IRT), mejoran las estimaciones sobre la capacidad del alumno cuando se incluyen más ítems en una prueba, lo cual también reduce el error de medición. Sin embargo, al utilizar el diseño de muestreo matriz, el TIMSS pudo usar un número menor de respuestas de cada alumno además de las características del trasfondo del alumno para imputar las puntuaciones de los alumnos, aunque no se esperaba que los alumnos respondieran a todos los ítems del TIMSS. El promedio de la escala del TIMSS está fijado en 500 con una desviación típica de 100. Usamos la puntuación de matemáticas de los alumnos en esta escala como nuestra variable dependiente.

Tabla 1. Variables del alumno y del centro de educación que se incluyen en el análisis

\begin{tabular}{|c|c|c|c|}
\hline & Variable & & Composición de la variable \\
\hline \multirow{5}{*}{$\begin{array}{l}\text { Trasfondo } \\
\text { del } \\
\text { Alumno }\end{array}$} & Edad & $\begin{array}{l}\text { - } 10 \text { a } 13 \text { años } \\
\text { - } 14 \text { a } 16 \text { años } \\
\text { - } 17 \text { y más }\end{array}$ & $\begin{array}{l}\text { La variable de la edad se definió de la } \\
\text { siguiente manera: }\end{array}$ \\
\hline & $\begin{array}{l}\text { Participación de } \\
\text { los padres }\end{array}$ & $\begin{array}{l}\text { - Rara vez } \\
\text { - Semanal } \\
\text { - Diaria }\end{array}$ & $\begin{array}{l}\text { - Mis padres me preguntan qué aprendí en el } \\
\text { centro; } \\
\text { - Hablo con mis padres sobre mis tareas } \\
\text { escolares; } \\
\text { - Mis padres se aseguran de que aparte } \\
\text { tiempo para hacer mis deberes; } \\
\text { - Mis padres comprueban si he hecho mis } \\
\text { deberes. }\end{array}$ \\
\hline & Acoso (bullying) & $\begin{array}{l}\text { - Casi cada semana } \\
\text { - Cada mes } \\
\text { - Casi nunca }\end{array}$ & $\begin{array}{l}\text { - Se burlaron de mí o me insultaron; } \\
\text { - Otros alumnos me excluyeron de juegos o } \\
\text { de actividades; } \\
\text { - Alguien propagó mentiras sobre mí; } \\
\text { - Otros alumnos me pegaron o me hicieron } \\
\text { daño; } \\
\text { - Otros alumnos me obligaron a hacer cosas } \\
\text { que no quería hacer. }\end{array}$ \\
\hline & $\begin{array}{l}\text { Si le gusta al } \\
\text { alumno aprender } \\
\text { matemáticas }\end{array}$ & $\begin{array}{l}\text { - No le gusta aprender } \\
\text { matemáticas } \\
\text { - Le gusta un poco aprender } \\
\text { matemáticas } \\
\text { - Le gusta aprender } \\
\text { matemáticas }\end{array}$ & $\begin{array}{l}\text { - Disfruto el aprendizaje de matemáticas; } \\
\text { - Me gustaría no tener que estudiar } \\
\text { matemáticas } \\
\text { - Las matemáticas son aburridas } \\
\text { - Aprendo muchas cosas interesantes en } \\
\text { matemáticas } \\
\text { - Me gustan las matemáticas } \\
\end{array}$ \\
\hline & $\begin{array}{l}\text { Cuánto valora las } \\
\text { matemáticas el } \\
\text { alumno }\end{array}$ & $\begin{array}{l}\text { - No las valora } \\
\text { - Algo de valor } \\
\text { - Valora las matemáticas }\end{array}$ & $\begin{array}{l}\text { - Es importante dominar las matemáticas. } \\
\text { - Aprender matemáticas me ayudará en la } \\
\text { vida diaria. } \\
\text { - Necesito las matemáticas para aprender } \\
\text { otras asignaturas escolares. } \\
\text { - Necesito dominar las matemáticas para } \\
\text { acceder a la universidad que deseo }\end{array}$ \\
\hline
\end{tabular}




\begin{tabular}{|c|c|c|c|}
\hline & \multirow{2}{*}{$\begin{array}{l}\text { El alumno está } \\
\text { seguro de sí } \\
\text { mismo en su } \\
\text { aprendizaje de } \\
\text { matemáticas }\end{array}$} & \multirow{2}{*}{\begin{tabular}{l|} 
\\
\\
- No está seguro \\
- Algo de seguridad \\
- Seguro en su aprendizaje \\
de matemáticas \\
\end{tabular}} & $\begin{array}{l}\text { - Necesito dominar las matemáticas para } \\
\text { obtener el trabajo que deseo } \\
\text { - Me gustaría tener un trabajo que incluya el } \\
\text { uso de las matemáticas. }\end{array}$ \\
\hline & & & $\begin{array}{l}\text { - } \begin{array}{l}\text { Generalmente me van bien las } \\
\text { matemáticas; }\end{array} \\
\text { - Las matemáticas son más difíciles para mí } \\
\text { que para muchos de mis compañeros; } \\
\text { - Las matemáticas no son uno de mis puntos } \\
\text { fuertes } \\
\text { - Aprendo matemáticas con facilidad } \\
\text { - Las matemáticas me confunden y me ponen } \\
\text { nervioso } \\
\text { - Soy bueno resolviendo problemas difíciles } \\
\text { de matemáticas } \\
\text { - Mi profesor piensa que puedo ser bueno en } \\
\text { matemáticas } \\
\text { Mi profesor me dice que soy bueno en } \\
\text { matemáticas } \\
\text { Las matemáticas son más difíciles para mí } \\
\text { que cualquier otra asignatura. }\end{array}$ \\
\hline \multirow{3}{*}{$\begin{array}{l}\text { Contexto } \\
\text { del centro } \\
\text { de } \\
\text { educación }\end{array}$} & $\begin{array}{l}\text { Especialización } \\
\text { en matemáticas }\end{array}$ & $\begin{array}{l}\text { - No hay especialización } \\
\text { en matemáticas } \\
\text { - Hay especialización en } \\
\text { matemáticas }\end{array}$ & $\begin{array}{l}\text { No hay especialización en matemáticas; Hay } \\
\text { especialización en matemáticas }\end{array}$ \\
\hline & $\begin{array}{l}\text { Escasez de } \\
\text { recursos }\end{array}$ & $\begin{array}{l}\text { - Afecta mucho } \\
\text { - Algo afectado } \\
\text { - No afecta }\end{array}$ & 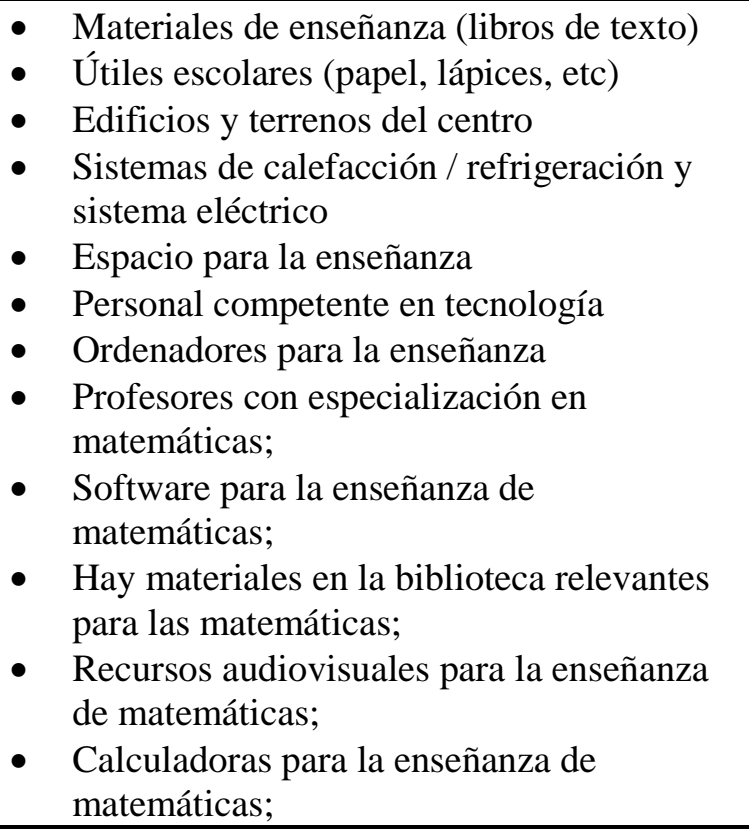 \\
\hline & $\begin{array}{l}\text { Condiciones } \\
\text { laborales del } \\
\text { Profesor }\end{array}$ & $\begin{array}{l}\text { - Problemas serios } \\
\text { - Problemas menores } \\
\text { - Casi ningún problema }\end{array}$ & $\begin{array}{l}\text { - El edificio del centro de educación necesita } \\
\text { importantes reparaciones; } \\
\text { - Las aulas están abarrotadas; } \\
\text { - Los profesores dan clase demasiadas horas } \\
\text { - Los profesores no tienen espacio de trabajo } \\
\text { adecuado } \\
\text { - Los profesores no tienen materiales ni } \\
\text { útiles adecuados para la enseñanza }\end{array}$ \\
\hline
\end{tabular}




\begin{tabular}{|c|c|c|}
\hline $\begin{array}{l}\text { Estatus } \\
\text { socioeconómico } \\
\text { del centro (SES) }\end{array}$ & $\begin{array}{l}\text { - Nivel bajo } \\
\text { - Nivel medio } \\
\text { - Nivel alto }\end{array}$ & $\begin{array}{l}\text { - Tamaño poblacional de las áreas que } \\
\text { rodean los centros; } \\
\text { - Porcentaje de alumnos provenientes de } \\
\text { hogares con ventajas o desventajas } \\
\text { económicas } \\
\text { - Nivel promedio de ingresos de los centros } \\
\text { en el entorno inmediato. }\end{array}$ \\
\hline
\end{tabular}

\section{Procedimiento: datos y variables del estudio}

El TIMSS Sudáfrica en el 2011 fue coordinado por el Consejo de investigación de ciencias humanas bajo el auspicio de la Asociación internacional para la evaluación del rendimiento educativo (International Association for the Evaluation of Educational Achievement (IEA). El TIMSS utiliza una metodología de muestreo sistemático estratificado en dos etapas de probabilidad proporcional al tamaño (PPS). En Sudáfrica, se tomaron muestras en un total de 298 centros y se seleccionó al azar una clase intacta del $9^{\circ}$ curso para incluirla en el estudio, lo que proporcionó el tamaño de muestra de 11969 alumnos. Los detalles sobre la metodología de muestreo se pueden obtener en la Guía internacional del usuario del TIMSS (Joncas y Foy, 2013) y también (Wu, 2010). El estudio incluyó la respuesta de los alumnos a preguntas de matemáticas y ciencias para evaluar su éxito en el aprendizaje de las matemáticas y de las ciencias en el 9no curso. El TIMSS también administró encuestas sobre el trasfondo al director del centro, al profesor y también al alumno. Nuestro análisis en este artículo se basa en el rendimiento de los alumnos en matemáticas. Ahora proporcionamos una descripción de las variables que usamos en nuestro análisis.

\section{Análisis estadístico}

Debido a la naturaleza jerárquica de los datos educativos se usarán técnicas de modelo multinivel con un paquete de software llamado Hierarchical Linear Modelling (modelos lineales jerárquicos (HLM)). El modelo multinivel funciona igual que las técnicas de regresión MCO (mínimos cuadrados ordinarios, en inglés, Ordinary Least Squares $(O L S)$ ) y aplican los mismos supuestos. Sin embargo, uno de los retos al usar los MCO para el análisis de los datos educativos es que no explica adecuadamente la varianza que es integral en cada uno de los niveles dentro de la jerarquía (Willms y Somer, 2001).

En el presente estudio se creó un modelo lineal jerárquico de 2 niveles (2-level HLM model) con las variables del trasfondo del alumno en el primer nivel y las variables del centro en el segundo nivel, incluidas todas en el modelo usando el centrado por la gran media (grand 
mean centering). Para el presente análisis se usó una ponderación muestral del alumno (TOTWGT) en el primer nivel (nivel del alumno); ésta es un compuesto de seis factores, concretamente: el peso del centro, de la clase y del alumno, así como un factor de ajuste para la no respuesta para cada uno de los pesos, del centro, de la clase y de los alumnos.

\section{El análisis multinivel}

Siguiendo a Raudenbusch y Bryk (2001), nuestro análisis multinivel comenzó con lo que a menudo se llama el modelo nulo. Esto es análogo al análisis de varianza (ANOVA) unidireccional tomando el logro de los alumnos de matemáticas como variable dependiente y el centro al que asisten como la variable independiente (variable de grupo). El análisis ANOVA nos permite probar la hipótesis de que la variabilidad entre centros de educación en cuanto al rendimiento es significativamente distinta de cero. Es más, el modelado inicial ANOVA de nivel multinivel también proporciona estimados de variación total en el logro de los alumnos en matemáticas, dividido entre la varianza entre centros $\left(\tau_{00}\right)$ y la varianza dentro del centro $\left(\sigma^{2}\right)$ que nos permitió calcular el coeficiente de correlación intraclase (ICC) el cual es la proporción de la variación total en los niveles de logros de los alumnos de matemáticas que pueden explicarse con la variación en los niveles de logros de los centros. El ICC para este estudio se calcula de la siguiente manera:

$$
\begin{gathered}
\rho=\frac{\tau 00}{\tau 00+\sigma 2} \text { (donde } \rho \text { es el ICC) } \\
=\frac{5242.61}{5242.61+3196.27} \\
=0,62=62 \%
\end{gathered}
$$

Esto significa que las diferencias en los alumnos de los centros explican alrededor del 62 por ciento de la variación total en sus niveles de logros en matemáticas. Las diferencias en las características del trasfondo de los alumnos dentro de los centros explican el resto de esta variación (alrededor del 38\%).

Las siguientes etapas del análisis intentaron aislar los factores específicos del centro y del trasfondo de los alumnos relacionados con el éxito de los alumnos en matemáticas. Esto implicaba probar la significación estadística del impacto de una serie de variables que describían las características del trasfondo del logro de los alumnos en matemáticas dentro de los centros. Se llevó a cabo un análisis similar para identificar una serie de características en el centro y en el aula que tienen impacto significativo sobre los niveles de logro en 
matemáticas de los centros. En nuestro modelo final, solo incluimos las variables del centro y del trasfondo de los alumnos con significación estadística al nivel alfa de 0,05. A continuación se encuentran las ecuaciones estadísticas usadas para estimar nuestros parámetros en el modelo final.

La ecuación a nivel multinivel final (dentro de y entre los centros)

$$
\begin{aligned}
& \text { Dentro del centro: } \left.\mathrm{Y}_{\mathrm{ij}}=\beta_{0 \mathrm{j}+}+\beta_{1 \mathrm{j}} \text { (MÁS17AÑS) }\right)_{1 \mathrm{ij}}+\beta_{2 \mathrm{j}}(\text { PartAdult })_{2 \mathrm{ij}}+\beta_{3 \mathrm{j}} \\
& (\text { Acoso })_{3 \mathrm{ij}}+\beta_{4 \mathrm{j}}(\text { GustMat })_{4 \mathrm{ij}}+\beta_{5 \mathrm{j}}(\text { ValMat })_{5 \mathrm{ij}}+\beta_{6 \mathrm{j}}(\text { ConfMat })_{6 \mathrm{ij}}+\mathrm{r}_{\mathrm{ij}}
\end{aligned}
$$

Entre centros: $\beta_{0 \mathrm{j}}=\gamma_{00}+\gamma_{01}(\text { SocEcCen })_{\mathrm{j}}+\gamma_{02}$ (Recur_Mat $)+\gamma_{03}($ EspeMat $)+$ $\gamma_{04}($ Condtr_Prof $)+\mu_{0 j}$

$$
\begin{aligned}
\beta_{1 j}=\gamma_{10}+\mu_{1 j} & \\
& \left\{\beta_{2 j}=\gamma 2_{0}+\mu_{2 j} \mid\right. \\
& \left\{\beta_{3 j}=\gamma_{30}+\mu_{3 j} \mid\right. \\
& \\
& \beta_{6 j}=\gamma_{60}+\mu_{6 j}
\end{aligned}
$$

Donde:

$\mathrm{Y}_{\mathrm{ij}}=$ la puntuación en matemáticas del alumno $i$ en el centro $j$;

$\beta_{0 \mathrm{j}}=$ el intercepto para el centro $\mathrm{j}$ (el nivel de logros promedio de matemáticas en el centro j);

$\beta_{1 \mathrm{j},}, \beta_{2 \mathrm{j},}, \beta_{3 \mathrm{j}}, \beta_{4 \mathrm{j}}, \beta_{5 \mathrm{j},}, \beta_{6 \mathrm{j}}=$ los coeficientes de regresión asociados a las variables de nivel 1. ;

$\gamma_{00}=$ Media nacional (gran media);

$\gamma_{01}, \gamma_{02}, \gamma_{03}, \gamma_{04}, \gamma_{05}, \gamma_{06}=$ los coeficientes de regresión asociados a las variables de nivel 2 ;

$\mu_{0 \mathrm{j}}=$ la desviación de la media del centro de la media nacional (total);

$\mu 0 \mathrm{j}=$ la desviación de la puntuación del alumno de la media del centro;

Los predictores del Nivel-1 son:

- MÁS17AÑS: Esta fue una variable dicotómica para comparar los alumnos de mayor edad con los alumnos que tienen la edad apropiada para el curso.

- PartAdult: La participación de un adulto en las tareas escolares del alumno.

- Acoso: Las respuestas del alumno al ser acosado en el centro de educación.

- GustMat, ValMat, ConfMat: La actitud de los alumnos hacia las matemáticas.

Los predictores del nivel-2 son:

- SocEcCen : El estatus socioeconómico del centro de educación; 
- Recur_Mat: Escasez de recursos de matemáticas;

- Espe_Mat: Especialización en matemáticas durante la calificación;

- Condtr_prof: Satisfacción del profesor con las condiciones laborales.

\section{Resultados}

Los resultados de los modelos multinivel se presentan en la Tabla 2, se explicarán en dos partes; el impacto de los factores significativos del trasfondo del alumno (nivel-1), seguidos por el impacto de los factores significativos del trasfondo del centro /profesor.

\section{El impacto de las características de trasfondo del alumno sobre su logro en matemáticas}

La Tabla2 muestra que la edad juega un rol importante en el rendimiento del alumno. En Sudáfrica, los niños comienzan su escolarización a la edad de 7 años por lo que, sin repetir curso, la edad esperada para los alumnos del $9^{\circ}$ curso es de 16 . Nuestro análisis agrupó los alumnos en edad apropiada o menor, y edad mayor (17 años y más) e hizo una estimación del impacto de la edad sobre el logro en matemáticas. La Tabla indica que los alumnos con 16 años o menores obtuvieron un promedio de 21 puntos más que los alumnos que tenían 17 años de edad o más.

Los resultados muestran también que la actitud de los alumnos hacia las matemáticas es extremadamente importante y está asociada a su rendimiento en matemáticas. Los alumnos que expresaron que les gustaban las matemáticas tuvieron mejor desempeño que los alumnos a los que no les gustaban las matemáticas, y los alumnos a los que les gustaban las matemáticas obtuvieron un promedio de 16,57 puntos más que los que dijeron que no les gustaban las matemáticas. Los alumnos que se sentían confiados en su aprendizaje de matemáticas tuvieron una puntuación promedio de 18,8 puntos más que los alumnos que dijeron que no se sentían confiados en el aprendizaje de matemáticas.

La participación de adultos en las tareas escolares de los alumnos está asociada al rendimiento en matemáticas. Los datos muestran que los adultos que se implican demasiado en las tareas escolares de sus hijos están asociados negativamente al rendimiento promedio del alumno. Los alumnos cuyos padres participan diariamente en los deberes tienen un desempeño promedio de 8,48 puntos menos que aquellos alumnos cuyos padres participan semanalmente. 
Otro hallazgo interesante es que los alumnos que dicen que casi nunca son acosados (bullied) tienen una puntuación promedio de 7,73 puntos más alta que los alumnos que decían que eran acosados diariamente.

\section{Factores escolares asociados a los efectos de la escuela}

Los alumnos que asisten a centros donde les enseña un profesor contento con sus condiciones laborales puntúan un promedio de 51,84 puntos más que los alumnos que asisten a centros donde el profesor está descontento con sus condiciones laborales.

La tabla 2 muestra que los alumnos de centros donde los profesores informaron que la escasez de recursos no era un problema puntuaron un promedio de 37,77 puntos más que los alumnos de centros donde los profesores dijeron que la escasez de recursos era un problema. Del modelo queda claro que los alumnos que asistían a centros donde el profesor estaba especializado en matemáticas puntuaban un promedio de 26,24 puntos más que los alumnos que asistían a centros donde el profesor no estaba especializado en matemáticas.

El estatus socioeconómico del centro (SES) es un factor significativo asociado al rendimiento y los alumnos de centros con un SES alto tenían mejor desempeño que los alumnos en centros con un SES bajo. Los alumnos que asisten a centros con un SES alto obtienen un promedio de 23 puntos más que los alumnos que asisten a centros con un SES bajo.

Tabla 2. Los modelos lineales jerárquicos (HLM)

\begin{tabular}{|l|l|l|l|}
\hline Variables & Modelo nulo & $\begin{array}{l}\text { Modelo 2-solo } \\
\text { con la variable de } \\
\text { trasfondo del } \\
\text { alumno }\end{array}$ & Modelo final (total) \\
\hline Intercepto & 344,00 & 343,88 & 349,76 \\
\hline Edad del alumno & & $-20,93$ & $-20,93$ \\
\hline La participación de adultos & & $-8,48$ & $-8,48$ \\
\hline Acoso (bullying) & & 7,73 & 7,73 \\
\hline $\begin{array}{l}\text { Cuánto le gustan al alumno } \\
\text { las matemáticas }\end{array}$ & 16,57 & 16,57 \\
\hline $\begin{array}{l}\text { Cuánto valora las } \\
\text { matemáticas el alumno }\end{array}$ & 6,70 & 6,70 \\
\hline Confianza del alumno & 18,80 & 18,80 \\
\hline $\begin{array}{l}\text { Estatus socioeconómico del } \\
\text { centro (SES) }\end{array}$ & & & 23,00 \\
\hline $\begin{array}{l}\text { Especialización en } \\
\text { matemáticas }\end{array}$ & & 26,24 \\
\hline
\end{tabular}




\begin{tabular}{|c|c|c|c|}
\hline Escasez de recursos & & & 37,77 \\
\hline Condiciones laborales & & & 51,84 \\
\hline \multicolumn{4}{|c|}{ Análisis de componente de varianza } \\
\hline Dentro del centro & 3196,27 & 2620,36 & 2620,23 \\
\hline Entre centros & 5242,61 & 5283,08 & 2753,95 \\
\hline $\begin{array}{l}\text { Porcentaje de la varianza } \\
\text { explicado }\end{array}$ & $62 \%$ & $33 \%$ & $49 \%$ \\
\hline
\end{tabular}

\section{Principales hallazgos y discusión}

Los resultados del modelo multinivel muestran que la varianza explicada por el rendimiento en matemáticas entre los centros es de 0,62 o 62\%. Esto significa que el centro donde estudia el alumno explica alrededor del 62 por ciento de la variación de su nivel de logro en matemáticas. Este porcentaje es ligeramente superior al 50\% que citaron Howie y Plomp (2003). Teniendo en cuenta que, en las últimas dos décadas el gobierno de Sudáfrica ha hecho un esfuerzo conjunto para mejorar la educación de calidad especialmente para los pobres y marginados, se esperaría una disminución en este porcentaje. Esto significa que las iniciativas políticas para mejorar los recursos en los centros pobres podrían no estar produciendo resultados significativos en el aprendizaje.

\section{Análisis dentro del centro - las características del alumno asociadas a la calidad}

La edad del alumno se incluyó en el estudio debido a que las investigaciones muestran que hay una relación entre la edad del alumno y el rendimiento (Grissom, 2004). En Sudáfrica la edad apropiada para el 9no curso es de entre los 14 y 16 años, y el análisis muestra que los alumnos con edades entre 14 y 16 tienen mejor rendimiento que los alumnos con 17 años o mayores. Parece que hay cierta contradicción en los resultados de las investigaciones respecto a la edad, ya que algunos estudios no encontraron diferencia significativa en la edad y el rendimiento del alumno (Kunje, Selemani-Meke, y Ogawa, 2009). Otros estudios encontraron una relación lineal significativa entre la edad y el rendimiento y que los alumnos mayores de esa edad tienen mejor rendimiento que los alumnos con la edad apropiada para el curso (Grissom, 2004). Sin embargo, Grissom afirma que esta relación lineal existe solamente en los cursos del los más pequeños y que, a medida que los alumnos avanzan hacia los cursos superiores, esta relación lineal se convierte en una negativa (Grissom, 2004). Los estudios muestran que la causa principal de edad excesiva que los alumnos repiten curso (Departamento de educación básica, 2011) . 
En el presente análisis se consideraron tres aspectos de actitudes hacia las matemáticas, concretamente: la confianza de los alumnos en el aprendizaje de las matemáticas, si a los alumnos les gustaba aprender matemáticas y finalmente, si valoraban las matemáticas. Los resultados muestran que todas las actitudes mencionadas tienen una asociación significativa con el rendimiento en matemáticas. Específicamente, los alumnos a los que les gustan las matemáticas, y que valoran y están confiados en el aprendizaje de matemáticas, tienen generalmente mejor rendimiento que los alumnos que tienen la actitud inversa hacia las matemáticas. Este hallazgo coincide con estudios realizados por la Organización para la Cooperación y el Desarrollo Económicos (OCDE) en el 2004 (Organisation for Economic Co-operation and Development, 2004) además de (Zan y Martino, 2007).

Los resultados también muestran una asociación entre la participación de los padres y el rendimiento del alumno en matemáticas lo cual contradice estudios realizados por Chiu y Ho (2006). Este análisis muestra que los padres que participan en las tareas escolares de los alumnos a diario tienen un efecto negativo en el rendimiento del alumno en matemáticas, algo que es apoyado por (Hoover Dempsey et al., 2005) quienes encontraron que los padres demasiado involucrados en las tareas escolares del alumno tienen una asociación negativa con el rendimiento del alumno. (Domina, 2005) encontró que la asociación entre la participación de los padres y el rendimiento del alumno se convierte en negativa o a veces insignificante, una vez están controlados el trasfondo de la familia y del centro. Otros estudios han encontrado que los padres son extremadamente significativos en las edades más tempranas pero se vuelven insignificantes según el alumno se hace mayor (Crosnoe, 2001).

El acoso (bullying) en el centro de educación parece ser un problema y a través de los años ha aumentado el porcentaje de alumnos que lo han sufrido. Los datos del TIMSS 2011 muestran que el 75\% de los alumnos ha sufrido acoso hasta cierto punto (Mullis, Martin, Foy, y Arora, 2012). El acoso (bullying) casi se ha duplicado en los últimos 11 años desde el ciclo del TIMSS 2002. El porcentaje de alumnos que dijeron que fueron acosados a menudo en el $2011(27,9 \%)$ es casi el doble de la cifra del 2002 (16\%) (Reddy, 2012). Los resultados del presente estudio muestran que los alumnos que dijeron que nunca o casi nunca habían sido acosados, obtuvieron puntuaciones superiores a las de aquellos alumnos que dijeron que eran acosados muy a menudo. Este resultado lo repiten (Nakamoto y Schwartz, 2010) quienes afirmaron que el acoso tiene un impacto negativo en el logro del alumno, lo que significa que 
los alumnos que experimentan acoso en el centro no tienen tan buen rendimiento como los alumnos que no son acosados.

\section{Características del centro de educación y el éxito de los alumnos en el aprendizaje}

Las condiciones laborales de los profesores pueden describirse adecuadamente con cuatro recursos escolares que, cuando se le proveen de forma adecuada al centro de educación, dan lugar a mejores condiciones laborales para el profesor (Johnson, 2006). Estos son: condiciones físicas adecuadas (edificios), un ambiente ordenado (el ambiente del centro), recursos didácticos (libros de texto y pizarras) y volumen de trabajo razonable (Johnson, 2006). Los resultados muestran que los alumnos a los que les enseña un profesor contento con sus condiciones laborales presentan un rendimiento mejor que los alumnos a los que les enseña un profesor que no está contento con sus condiciones laborales. Los resultados los repiten investigadores tales como (Rosenholtz y Simpson, 1990) y (Firestone y Pennell, 1993)

Los resultados muestran que los alumnos cuyos profesores se especializaron en matemáticas o en la enseñanza de matemáticas obtuvieron puntuaciones superiores que los alumnos a los que les enseñaban profesores sin especialización. Este hallazgo está en línea con una investigación previa que muestra que el mero hecho de tener el título de educación superior no es suficiente para asegurar puntuaciones óptimas en las pruebas de los alumnos, sino que es vital asegurarse de que los profesores responsables de la enseñanza de matemáticas estén también especializados en el área (Goldhaber y Brewer, 2000)y también (Rowan, Chiang, y Miller, 1997).

La escasez de recursos didácticos fue una escala calculada e incluía recursos del centro tales como libros de texto de matemáticas, material de oficina, el edificio del centro, y las aulas. Los resultados muestran que los alumnos a los que les enseñaba un profesor que experimentaba poca o ninguna escasez tenían mejor rendimiento que los alumnos de centros donde se experimentaba escasez. Este hallazgo lo apoyan (Fuller y Clarke, 1994) sugiriendo que la disponibilidad de recursos educativos tenía la relación significativa más positiva con el rendimiento del alumno. Sin embargo, la situación en los países desarrollados y en los países en desarrollo es muy diferente, donde factores relacionados con las variables de la organización del centro y la instrucción (Scheerens, 2000) son más importantes que la disponibilidad del material didáctico. 
Se encontró que el estatus socioeconómico del centro está asociado con el rendimiento en matemáticas del alumno en cuanto a que los alumnos que asisten a centros con SES alto se desempeñaban mejor que sus homólogos. Esto va en la línea de los hallazgos de (Willms y Somer, 2001) y de (X. Ma y Xu, 2004) y (X. Ma y Klinger, 2000).

\section{Observaciones finales}

El desarrollo de un sistema educativo eficaz que provea oportunidades para que todos los alumnos tengan éxito en los centros ha sido uno de los objetivos importantes de países de todo el mundo. Este objetivo está impulsado en gran medida por la opinión de que un sistema educativo exitoso donde se educa a la mayoría de los ciudadanos conduce a una economía próspera, a cohesión social y a calidad de vida. El desarrollo de un sistema educativo eficaz es un proceso complejo que requiere valoración continua y las evaluaciones del proceso educativo y sus resultados. A nivel internacional, las valoraciones educativas a gran escala tales como el Estudio de las tendencias en matemáticas y ciencias (el TIMSS, Trends in International Mathematics and Science Study) proveen oportunidades para que los sistemas educativos realizan "evaluaciones exhaustivas de última generación” del logro de los alumnos en relación con sus experiencias de escolarización y de aprendizaje. El análisis de estos datos exige a menudo procedimientos estadísticos complejos como modelos multinivel que nos permiten evaluar el efecto del centro de educación - la medida en que los centros inciden en el éxito de los alumnos en cuanto a la escolarización. En Sudáfrica, son raros los análisis de los efectos de la escuela que usan los modelos multinivel.

Nuestro artículo, que usa los datos del 2011 del TIMSS de Sudáfrica, usa modelos multinivel para identificar los indicadores más importantes de los centros con éxito de Sudáfrica. El análisis multinivel indica que el rendimiento de los alumnos es superior en los centros que cuentan con los recursos adecuados, donde los profesores están satisfechos con sus condiciones laborales y están especializados en su área de enseñanza. Dentro de los centros, es extremadamente importante la actitud de los alumnos hacia las matemáticas porque aquellos a los que les gustan las matemáticas, los que valoran las matemáticas y se sienten confiados en el aprendizaje de matemáticas tienden a ser más exitosos. 
Los hallazgos demuestran la necesidad de que el gobierno de Sudáfrica continúe mejorando el ambiente de aprendizaje en los centros con menos recursos y que también anime a los profesores a hacer más divertido el aprendizaje de matemáticas. Hace aproximadamente dos décadas, Sudáfrica se convirtió en un país democrático alejándose del sistema educativo del apartheid donde el acceso a la educación de calidad se determinaba según el color de la piel, hacia una educación de calidad garantizada constitucionalmente para todos los alumnos sin importar sus características de trasfondo. El esfuerzo político significa mayormente una mejora de los recursos de los centros localizados en comunidades pobres. Nuestros hallazgos sugieren la necesidad de incluir iniciativas políticas para ayudar a los alumnos a desarrollar una actitud positiva y confianza propia en el aprendizaje de matemáticas.

\section{References}

Crosnoe, R. (2001). Academic orientation and parental involvement in education during high school. Sociology of Education, 74, 210-230.

Department of Basic Education. (2011). Report on dropout and learner retention strategy to protfolio committee on education. Consultado, 2013 desde ehttp://www.education.gov.za/LinkClick.aspx?fileticket=jcSsY0rHcME\%3D\&tabid=422 $\underline{\text { mid }=1261}$

Domina, T. (2005). Leveling the home advantage: Assessing the effectiveness of parental involvement in elementary school. Sociology of Education, 78(3), 233-249. doi: $10.1177 / 003804070507800303$

Firestone, W. A., \& Pennell, J. R. (1993). Teacher commitment, working conditions, and differential incentive policies. Review of Educational Research, 63(4), 489-525. doi: 10.3102/0034654306300448

Foy, P. (2013). TIMSS and PIRLS 2011 user guide for the fourth grade combined international database.

Foy, P., Brossman, B., \& Galia, J. (2011). Scaling the TIMSS and PIRLS 2011 achievement data. TIMSS and PIRLS Methods and Procedures. Zugriff Am, 29, 2013.

Fuller, B., \& Clarke, P. (1994). Raising school effects while ignoring culture? Local conditions and the influence of classroom tools, rules, and pedagogy. Review of Educational Research, 64(1), 119-157. doi: 10.3102/00346543064001119 
Goldhaber, D. D., \& Brewer, D. J. (2000). Does teacher certification matter? High school teacher certification status and student achievement. Educational Evaluation and Policy Analysis, 22(2), 129-145. doi: 10.3102/01623737022002129

Grissom, J. B. (2004). Age and achievement. Education Policy Analysis Archives, 12(49). http://dx.doi.org/10.14507/epaa.v12n49.2004

Hoover Dempsey, K. V., Walker, J. M. T., Sandler, H. M., Whetsel, D., Green, C. L., Wilkins, A. S., \& Closson, K. (2005). Why do parents become involved? Research findings and implications. The Elementary School Journal, 106(2), 105-130. DOI: $10.1086 / 499194$

Howie, S. J., \& Plomp, T. (2003). Language proficiency and contextual factors influencing secondary students' performance in mathematics in South Africa. ERIC.

Hungi, N., \& Thuku, F. W. (2010). Variations in reading achievement across 14 Southern African school systems: Which factors matter? International Review of Education, 56(1), 63-101. doi:10.1007/s11159-009-9148-х

Johnson, S. M. (2006). The workplace matters: Teacher quality, retention and effectiveness National Education Association Washington, DC. Consultado desde http://files.eric.ed.gov/fulltext/ED495822.pdf

Joncas, M., \& Foy, P. (2013). Sample design in TIMSS and PIRLS. In M. O. Martin, \& I. V. S. Mullis (Eds.), Methods and procedures in TIMSS and PIRLS (pp. 1-21). Lynch School of Education, Boston College: TIMSS and PIRLS International Study Centre.

Kunje, D., Selemani-Meke, E., \& Ogawa, K. (2009). An investigation of the relationship between school and pupil characteristics and achievement at the basic education level in Malawi. Journal of International Cooperation in Education, 12(1)

Lee, V. E., Zuze, T. L., \& Ross, K. N. (2005). School effectiveness in 14 sub-saharan African countries: Links with 6th graders' reading achievement. Studies in Educational Evaluation, 31(2), 207-246. DOI:10.1016/j.stueduc.2005.05.011

Levin, H. M. (2001). High-stakes testing and economic productivity. Raising Standards Or Raising Barriers, 39-50.

Ma, X., \& Klinger, D. A. (2000). Hierarchical linear modelling of student and school effects on academic achievement. Canadian Journal of Education/Revue Canadienne De l'Education, , 41-55. DOI: 10.2307/1585867

Ma, X., \& Xu, J. (2004). Determining the causal ordering between attitude toward mathematics and achievement in mathematics. American Journal of Education, 110(3), 256-280. DOI: $10.1086 / 383074$ 
Mullis, I. V. S., Martin, M. O., Foy, P., \& Arora, A. (2012). Chapter 6: School climate. TIMSS 2011 international results in mathematics (pp. 247) TIMSS \& PIRLS International Study Center and International Association for the Evaluation of Educational Achievement (IEA).

Nakamoto, J., \& Schwartz, D. (2010). Is peer victimization associated with academic achievement? A Meta-analytic review. Social Development, 19(2), 221-242. DOI: 10.1111/j.1467-9507.2009.00539.x

Organisation for Economic Co-operation and Development. (2004). Learning for tomorrow's world: First results from PISA 2003 OECD Publishing. DOI: 10.1787/9789264006416en

Reddy, V. (2012). Towards equity and excellence. highlights from TIMSS 2011. The south african perspective. Manuscrito sin publicar. Consultado desde http://www.hsrc.ac.za/uploads/pageContent/2929/TIMSSHighlights2012Dec7final.pdf

Rosenholtz, S. J., \& Simpson, C. (1990). Workplace conditions and the rise and fall of teachers' commitment. Sociology of Education, 241-257. http://dx.doi.org/10.2307/2112873

Rowan, B., Chiang, F., \& Miller, R. J. (1997). Using research on employees' performance to study the effects of teachers on students' achievement. Sociology of Education, 256-284.

Rumberger, R. W., \& Palardy, G. J. (2004). Multilevel models for school effectiveness research. Handbook of Quantitative Methodology for the Social Sciences. DOI:10.1080/0924345960070101

Scheerens, J. (2000). Improving school effectiveness Unesco, International Institute for Educational Planning.

Willms, J. D., \& Somer, M. (2001). Family, classroom, and school effects on childrens educational outcomes in Latin America. School Effectiveness and School Improvement, 12(4), 409-445. DOI:10.1076/sesi.12.4.409.3445

Wu, M. (2010). Measurement, sampling, and equating errors in Large-Scale assessments. Educational Measurement: Issues and Practice, 29(4), 15-27.

Zan, R., \& Martino, P. (2007). Attitudes towards mathematics: Overcoming positive/negative dichotomy. The Montana Mathematics Enthusiasts, 157-168. http://dx.doi.org/10.1155/2012/876028 